\title{
BRITE-Constellation: Nanosatellites for precision photometry of bright stars
}

\author{
W. W. Weiss ${ }^{1 \dagger}$, A. F. J. Moffat ${ }^{2 \dagger}$, A. Schwarzenberg-Czerny ${ }^{3 \dagger}$, \\ O. F. Koudelka ${ }^{4 \dagger}$, C. C. Grant ${ }^{5}$, R. E. Zee ${ }^{5}$, R. Kuschnig ${ }^{\dagger \dagger}$,
} St. Mochnacki ${ }^{6 \dagger}$, S. M. Rucinski ${ }^{6 \dagger}$, J. M. Matthews ${ }^{7 \dagger}$, P. Orleański ${ }^{8 \dagger}$, A. A. Pamyatnykh ${ }^{3 \dagger}$, A. Pigulski ${ }^{9 \dagger}$, J. Alves $^{1 \dagger}$, M. Guedel $^{1 \dagger}$, G. Handler ${ }^{3 \dagger}$, G. A. Wade ${ }^{10 \dagger}$, A. L. Scholtz ${ }^{11}$, and the CCD Tiger Team $\ddagger$

\footnotetext{
${ }^{1}$ University of Vienna, Institute for Astrophysics, Tuerkenschanzstr. 17, 1180 Vienna, Austria; email: werner.weiss@univie.ac.at

${ }^{2}$ Dept. de physique, Université de Montréal, Canada; ${ }^{3}$ Copernicus Astronomical Center, Warsaw, Poland; ${ }^{4}$ Graz University of Technology, Graz, Austria; ${ }^{5}$ Space Flight Laboratory, University of Toronto, Canada; ${ }^{6}$ Dept. of Astronomy and Astrophysics, University of Toronto, Canada; ${ }^{7}$ Dept. of Physics and Astronomy, University of British Columbia, Canada; ${ }^{8}$ Space

Research Center of the Polish Academy of Sciences, Warsaw, Poland; ${ }^{9}$ Astron. Institute, University of Wrocław, Poland; ${ }^{10}$ Dept. of Physics, Royal Military College of Canada, Ontario, Canada; ${ }^{11}$ Institute of Telecommunications, Vienna University of Technology, Austria
}

Abstract. BRITE-Constellation (where BRITE stands for BRIght Target Explorer) is an international nanosatellite mission to monitor photometrically, in two colours, brightness and temperature variations of stars brighter than $V \approx 4$, with precision and time coverage not possible from the ground. The current mission design consists of three pairs of $7 \mathrm{~kg}$ nanosats (hence "Constellation") from Austria, Canada and Poland carrying optical telescopes $(3 \mathrm{~cm}$ aperture) and CCDs. One instrument in each pair is equipped with a blue filter; the other, a red filter. The first two nanosats (funded by Austria) are UniBRITE, designed and built by UTIASSFL (University of Toronto Institute for Aerospace Studies-Space Flight Laboratory) and its twin, BRITE-Austria, built by the Technical University Graz (TUG) with support of UTIASSFL. They were launched on 25 February 2013 by the Indian Space Agency, under contract to the Canadian Space Agency.

Each BRITE instrument has a wide field of view ( $\approx 24$ degrees), so up to 15 bright stars can be observed simultaneously in $32 \times 32$ sub-rasters. Photometry (with reduced precision but thorough time sampling) of additional fainter targets will be possible through on-board data processing. A critical technical element of the BRITE mission is the three-axis attitude control system to stabilize a nanosat with very low inertia. The pointing stability is better than 1.5 arcminutes rms, a significant advance by UTIAS-SFL over any previous nanosatellite.

BRITE-Constellation will primarily measure p- and g-mode pulsations to probe the interiors and ages of stars through asteroseismology. The BRITE sample of many of the brightest stars in the night sky is dominated by the most intrinsically luminous stars: massive stars seen at all evolutionary stages, and evolved medium-mass stars at the very end of their nuclear burning phases (cool giants and AGB stars). The Hertzsprung-Russell diagram for stars brighter than mag $V=4$ from which the BRITE-Constellation sample will be selected is shown in Fig. 1. This sample falls into two principal classes of stars:

(1) Hot luminous H-burning stars (O to F stars). Analyses of OB star variability have the potential to help solve two outstanding problems: the sizes of convective (mixed) cores in massive stars and the influence of rapid rotation on their structure and evolution.

(2) Cool luminous stars (AGB stars, cool giants and cool supergiants). Measurements of the

$\dagger$ Member of the BRITE-Constellation Executive Science Team (BEST).

$\ddagger$ M. Chaumont, C. Grant, J. Lifshits, A. Popowicz, M. Rataj, P. Romano, M. Unterberger, R. Wawrzaszek, T. Zawistowski \& BEST. 


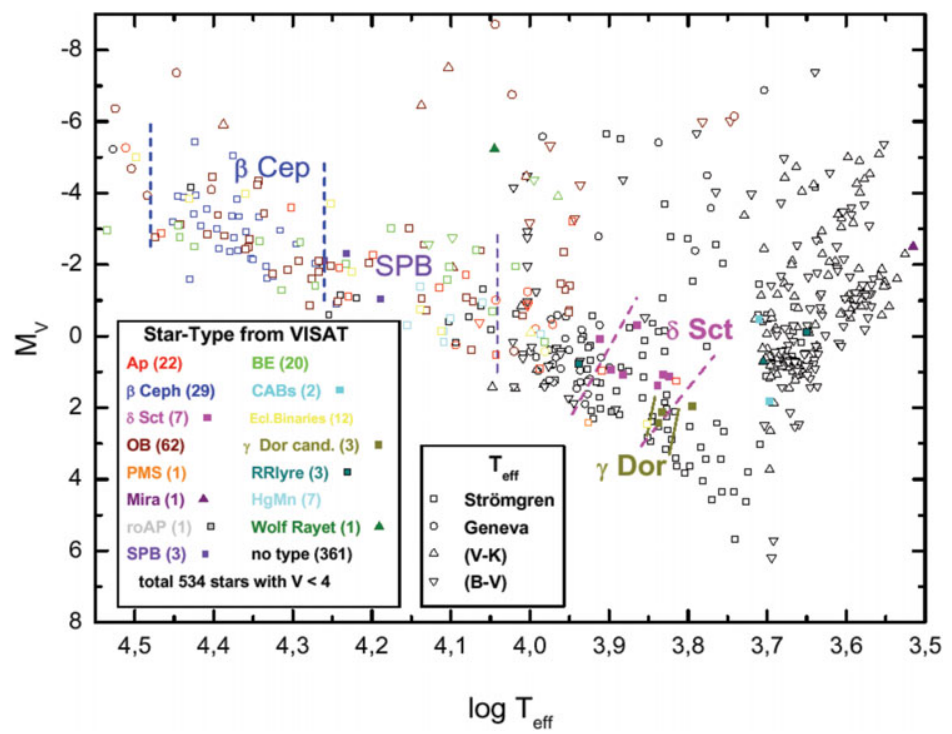

Figure 1. H-R diagram for stars brighter than mag $V=4$ with instability strips indicated.

time scales involved in surface granulation and differential rotation will constrain turbulent convection models.

Mass loss from these stars (especially the massive supernova progenitors) is a major contributor to the evolution of the interstellar medium, so in a sense, this sample dominates cosmic "ecology" in terms of future generations of star formation. The massive stars are believed to share many characteristics of the lower mass range of the first generation of stars ever formed (although the original examples are of course long gone).

BRITE observations will also be used to detect some Jupiter- and even Neptune-sized planets around bright host stars via transits, as expected on the basis of statistics from the Kepler exoplanet mission. Detecting planets around such very bright stars will greatly facilitate their subsequent characterization. BRITE will also use surface spots to investigate stellar rotation.

The following Table summarizes launch and orbit parameters of BRITE-Constellation components.

\begin{tabular}{|c|c|c|c|c|c|c|c|}
\hline Designation & Name & $\mathrm{F}$ & Vehicle & $\begin{array}{l}\text { Launch } \\
\text { date }\end{array}$ & $\begin{array}{l}\text { Orbit } \\
\text { km }\end{array}$ & $\begin{array}{c}\text { Descending } \\
\text { node }\end{array}$ & $\begin{array}{c}\text { Drift } \\
\text { min }\end{array}$ \\
\hline \multicolumn{8}{|c|}{ Owner: Austria } \\
\hline BRITE-A & BRITE-Austria & $B$ & PSLV-21 & 25 Feb 2013 & 800 & 18:00 & 0 \\
\hline BRITE-U & UniBRITE & $R$ & PSLV-21 & 25 Feb 2013 & circular & $18: 00$ & 0 \\
\hline \multicolumn{8}{|c|}{ Owner: Canada } \\
\hline BRITE-C1 & Toronto & & DNEPR & Q3-4/2014 & $629 \times 577$ & $10: 30$ & 40 \\
\hline BRITE-C2 & Montréal & & DNEPR & Q3-4/2014 & $629 \times 577$ & $10: 30$ & 40 \\
\hline \multicolumn{8}{|c|}{ Owner: Poland } \\
\hline $\begin{array}{l}\text { BRITE-P1 } \\
\text { BRITE-P2 }\end{array}$ & $\begin{array}{c}\text { Lem } \\
\text { Heweliusz }\end{array}$ & $\begin{array}{l}B \\
R\end{array}$ & $\begin{array}{c}\text { DNEPR } \\
\text { China LM-4 }\end{array}$ & $\left|\begin{array}{c}21 \text { Nov. } 2013 \\
2014\end{array}\right|$ & $\begin{array}{l}600 \times 900 \\
\mathrm{SSO} / 630\end{array}$ & $10: 30$ & 100 \\
\hline
\end{tabular}

The full version of this paper describing in more detail BRITE-Constellation will be published separately in a journal. The symposium presentation is available at http://iaus301.astro.uni.wroc.pl/program.php

Keywords. space vehicles, instrumentation: photometric, stars: general, interiors, oscillations 